\title{
Multicast Tree Formation and Analysis the Performance in AODV Protocol
}

\author{
Gurpreet Singh Bagga \\ Student \\ Department of electronics and communication \\ Lovely Professional University \\ Phagwara, Punjab.
}

\author{
Mritunjay Kumar Rai \\ Associate Professor \\ Department of electronics and communication \\ Lovely Professional University \\ Phagwara, Punjab.
}

\begin{abstract}
MANET applications has great benefits from multicast and apply in the fields where multicast performs effectively and efficiently. Multicasting is a technique which provides an important service for optimizing bandwidth consumption, throughput, and delay of distributed systems when number of users take participate in communication session simultaneously. On- demand routing protocols is used for the route establishment from source to destination only when it is required. AODV and DSR are the reactive routing protocols which uses flooding approach for establishing the route from source to destination. In this paper we propose an algorithm in which multicast tree is computed in network with the nodes having limited multicast capability and the problem of flooding is overcome with the same technique. The performance differentials are analyzed using varying simulation time. These simulations are carried out using the ns-2 network simulator. The simulation results shows that proposed technique is better than existing technique in terms of packet loss, delay and throughput.
\end{abstract}

Keywords: MANET, AODV, DSDV, divides and Conquer scheme, RREQ, RREP.

\section{INTRODUCTION}

A network is a group of two or more computer systems which linked together. It is mode of exchange of information to communicate with one another. Ad hoc wireless networks are the decentralized type of network. In ad hoc network resource management and routing between source to destination is done using distributed approach in which coordination of each node requires to enable communication. ad hoc network is used in urgent situation. No fixed infrastructure like base station is needed in ad hoc network. Ad hoc use multi hop technique for communication [1].

There are two types of classification available in adhoc networks which are as following:

\section{Single-hop \\ II. Multi-hop}

Single-hop: In this hop nodes are in direct communication and both nodes are in range of each others. The chances of link failure are more in this hop[1].

Multi-hop: In this hop nodes are communicate with the help of internal nodes not directly. To reach from source the destination intermediate nodes participate.
There different types of adhoc network available. MANET is a mobile adhoc network. It is self-configuring network which is infrastructure less in nature. In Manet different mobiles are connected through wireless link. Each mobile are free to move i.e. no central controller available [5,6]. A wireless sensor network is collections of sensing device that can be wirelessly communicate. Each device is capable of talk to its peer, sense, process. It is centralized system. It is inexpensive to install and no wiring is required for data transfer [3].Wireless mesh network is a communication network made up of radio nodes which are ordered in a mesh topology. Wireless mesh networks made up of gateways, mesh routers, mesh clients and. The mesh clients are may be laptops, cell phones and other wireless devices. The traffic is forwarded by mesh routers to and from the gateways but not connect to the internet. An ad hoc network is a wireless network describe by the nonexistence of a centralized and fixed infrastructure. The absence of an infrastructure in ad hoc networks poses great challenges in the functionality of these networks. Therefore, we refer to a wireless ad hoc network with mobile nodes as a Mobile Ad Hoc Network. In a MANET, mobile nodes have the capability to accept and route traffic from their intermediate nodes towards the destination, i.e., they can act as both routers and hosts. More frequent connection breaking and re-associations place an energy constraint on the mobile nodes.

\subsection{Routing Protocols in MANET}

Routing protocol indicates that how to communicate with the help of routers. It shares information among intermediate nodes then with the whole network. It helps to search shortest path from source to destination. There are mainly two types of routing protocol available [4].

\subsubsection{Proactive Routing Protocol}

In Proactive protocol all nodes maintain fresh list of the route and their destination from source in the form of tables. Routing information is flooded in whole network and path finding algorithm applies when source wants path to destination. All the nodes are updating their table regularly. If the topology frequently changes than update information propagate to each node and table is updated [10].

\section{DESTINATION SEQUENCE DISTANCE VECTOR (DSDV).}

It is table driven protocol in which route to all the destinations are present at each node. At a regular interval of time tables are exchanged between the neighbors. Every node maintains a routing table that have lists of all available destinations, the assigned sequence number by the destination node and the 
number of hops to reach the destination. The sequence number is used to distinguish old routes from new ones and to avoid the loops formation. The periodically stations transmit their routing tables to their instant neighbors. If a significant amount of change has occurred in its table from the last update then station also transmits its routing table. If two routes have the same sequence number then the route with the shortest route is used. DSDV does not support multi-path routing. It provides shortest path of good quality, trust worthy and cooperative [11].

Tables are update with two types, incremental and full dump.

Incremental update is used when node do not observe changes in local topology.

Full dump is used when there is change in local topology.

\subsubsection{Reactive Routing Protocol}

It is on-demand routing protocol. It finds the path only when it is required. These protocols do not maintain network topology information and not exchange information periodically. It maintain table only on demand. Route discovery process is followed to find path. These are bandwidth efficient protocol. In this protocol route request packet is sent across the network and route reply message comes from intermediate nodes. Route error message is issued by nodes if link is break. Its disadvantage is when flooding is excessive then congestion occurs [2].

\section{AD HOC ON-DEMAND DISTANCE VECTOR}

It is an on demand routing protocol means path from source to destination is created only when it is required. It uses the destination sequence number to find up-to-date path to destination and to find most recent path. In AODV source and intermediate node stores the next hop information [3]. If destination sequence number of current received packet is greater than previous number only then node updates itself.

Three messages define in AODV: Route Requests (RREQ), Route Errors (RERR) and Route Replies (RREP). These messages are used to discover the route and maintain routes across the network from source node to destination [2].

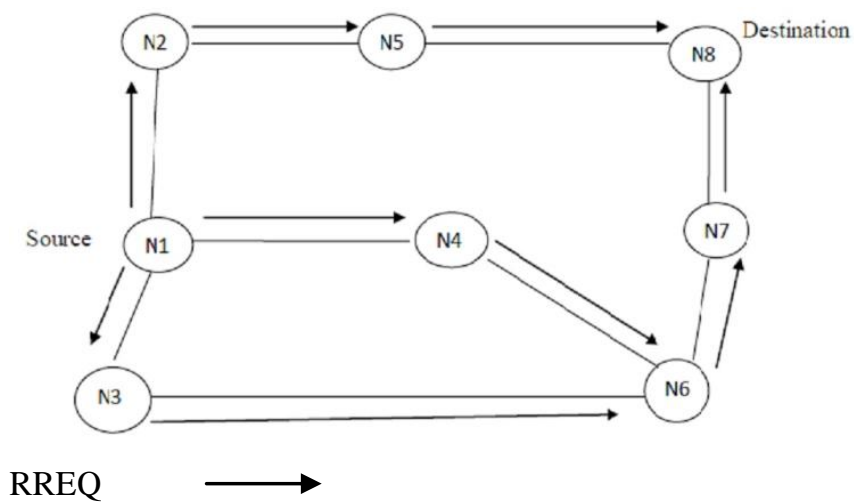

Fig 1(a): sending RREQ packets

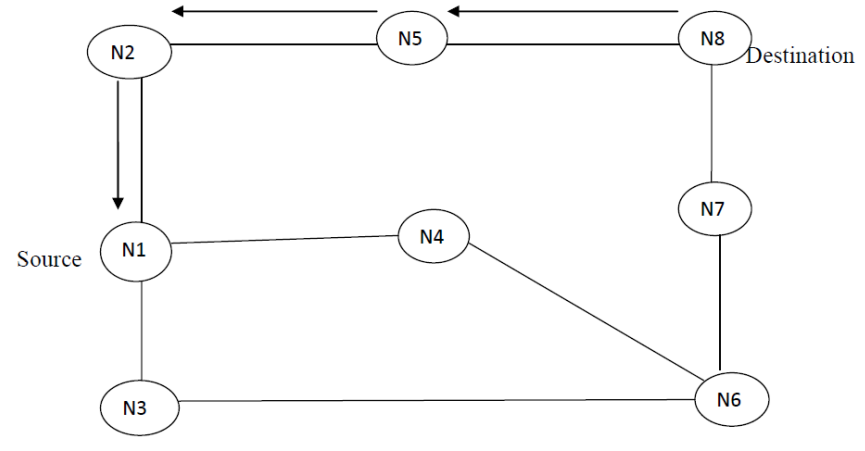

RREP

Fig 1(b): Best path with minimum Hop Count

(see Figure 1(a)) N1 is the source nodes which broadcasts the packets to its neighbor nodes with RREQ message. Then these nodes further forwards packets to its neighbor until the destination find outs [12] after reaching to the destination intermediate nodes reply with route reply message RREP.

There are some issues of this protocol also. To overcome these problem multicasting protocols are introduced.

\section{DIVIDE AND CONQUER SCHEME}

Divide and Conquer scheme [7] is based on static clustering and minimum distance based multicasting node selection. Network area is logically divided into small regions (clusters). The beauty of this technique is the formation of square and rectangular regions, which divides the network field into small regions, as a result the communication distance for intra cluster and inter cluster reduces. There are three steps which are used to divide the region.

\subsection{Formation of regions:}

I. In first step, network is divided into $n$ equal distant concentric squares. For simplicity, take $n=3$ here therefore, network is divided into three equal distance concentric squares: Internal square (Is), Middle square (Ms) and Outer square (Os).

II. BS is located in the centre of network field therefore; its coordinates are taken as reference point for formation of concentric squares.

III. Division of network field into concentric squares can be obtained from following equations:

IV. Coordinates of top right corner of Is, $\operatorname{Tr}(\mathrm{Is})$

V. $\quad \operatorname{Tr}(\mathrm{Is})=(\mathrm{Cp}(\mathrm{x})+\mathrm{d}, \mathrm{Cp}(\mathrm{y})+\mathrm{d})$

VI. Coordinates of bottom right corner of Is, $\mathrm{Br}$ (Is)

VII. $\quad \operatorname{Br}(\mathrm{Is})=(\mathrm{Cp}(\mathrm{x})+\mathrm{d}, \mathrm{Cp}(\mathrm{y})-\mathrm{d})$

VIII. Coordinates of top left corner of Is, Tl(Is)

IX. $\quad \mathrm{Tl}(\mathrm{Is})=(\mathrm{Cp}(\mathrm{x})-\mathrm{d}, \mathrm{Cp}(\mathrm{y})+\mathrm{d})$

X. Coordinates of bottom left corner of Is, Bl(Is)

XI. $\quad \mathrm{Bl}(\mathrm{Is})=(\mathrm{Cp}(\mathrm{x})-\mathrm{d}, \mathrm{Cp}(\mathrm{y})-\mathrm{d})$

XII. Where, $d$ is the factor of distance from center of network to boundary of Is value of $\mathrm{d}$ for Ms and Os increases with a multiple of 2 and 3 respectively. If there are $n$ number of concentric squares then the coordinates of nth square can be found, $\mathrm{Sn}$ from the following equations.

XIII. $\quad \operatorname{Tr}(\operatorname{Sn})=(\mathrm{Cp}(\mathrm{x})+\mathrm{dn}, \mathrm{Cp}(\mathrm{y})+\mathrm{dn})$

XIV. $\quad \operatorname{Br}(\mathrm{S} n)=(\mathrm{Cp}(\mathrm{x})+\mathrm{dn}, \mathrm{Cp}(\mathrm{y})-\mathrm{dn})$

XV. $\quad \operatorname{Tl}(S n)=(C p(x)-d n, C p(y)+d n)$ 
XVI.

$\mathrm{Bl}(\mathrm{S} \mathrm{n})=(\mathrm{Cp}(\mathrm{x})-\mathrm{dn}, \mathrm{Cp}(\mathrm{y})-\mathrm{dn})$

XVII. In the second step, the area is divided into two concentric squares into equal area quadrilaterals; latter is named as Corner Regions(CR) and NonCorner Regions(NCR).

\subsection{Multicasting Node}

Divide-and-Rule protocol considers multi-hop communication for inter-cluster communication. As it is assumed that $n=3$ therefore, inter-cluster communication is performed at two levels, that is, at primary level and at secondary level. $\mathrm{CH}$ selection follows, following approach.

\subsubsection{Primary Level Cluster Head}

Primary level $\mathrm{CH}$ selection follows the sequence:

I. Nodes whose co-ordinates lie in (Is) are nearer to BS therefore, they send data directly to BS [8]. As clusters are static, therefore one $\mathrm{CH}$ is selected in each NCR.

II. Midpoint of each NCR is considered as reference point for selection of $\mathrm{CH}$ in that region.

III. Nearest node from central reference point is selected as $\mathrm{CH}$.

IV. Next nearest node from the reference point is selected as $\mathrm{CH}$ for next round and so on.

\subsubsection{Secondary Level Cluster Head}

Steps followed in selection of secondary level $\mathrm{CHs}$ are:

I. $\mathrm{CHs}$ in Os regions send data to $\mathrm{CHs}$ of exactly one level above adjacent region's $\mathrm{CH}$. These $\mathrm{CHs}$ are also known as secondary level $\mathrm{CHs}$,

II. Secondary level $\mathrm{CHs}$ aggregate their own cluster nodes data and, data of the primary level $\mathrm{CH}$ then, transmit data to BS [8].

\subsection{Protocol Operation}

In setup phase BS divides the network field into small regions, on the bases of their co-ordinates. Is nodes send data directly to BS. In each region one $\mathrm{MN}$ is selected per round. MNs of Os regions, select front neighboring MNs of Ms regions as their next hop MN. Nodes of CR selects, BS or neighboring $\mathrm{MNs}$ as their $\mathrm{MN}$, based on minimum distance. If a tie occurs, for a node of CR, in selection of MN from its neighboring regions than, it is resolved by selecting the $\mathrm{MN}$ with greater residual energy. In steady state phase each node send its data to $\mathrm{MN}$ in its allocated time slot. Primary level MNs send aggregated data to their respective secondary level MNs. Secondary level MNs then, aggregate all collected data and forward it to BS.

\section{PROPOSED METHODOLOGY}

The route from the source to destination is established with the Proactive and reactive routing protocols. The Proactive routing protocols are those which store the route matrix and with the use of these matrices reliable route is established between source and destination. In the MANET, it is very difficult to maintain such matrices due to its self configuring nature. In such, case another type of routing protocol, .i.e. Reactive routing protocols is used for the route establishment. In reactive routing protocols routes are established between source and destination only when required. AODV and DSR are two most common type of protocols which are used for the route establishment. In AODV routing protocol dijkstra's algorithm is been used,as compared to DSR it is single source algorithm and belmans ford algorithm is used for route establishment.

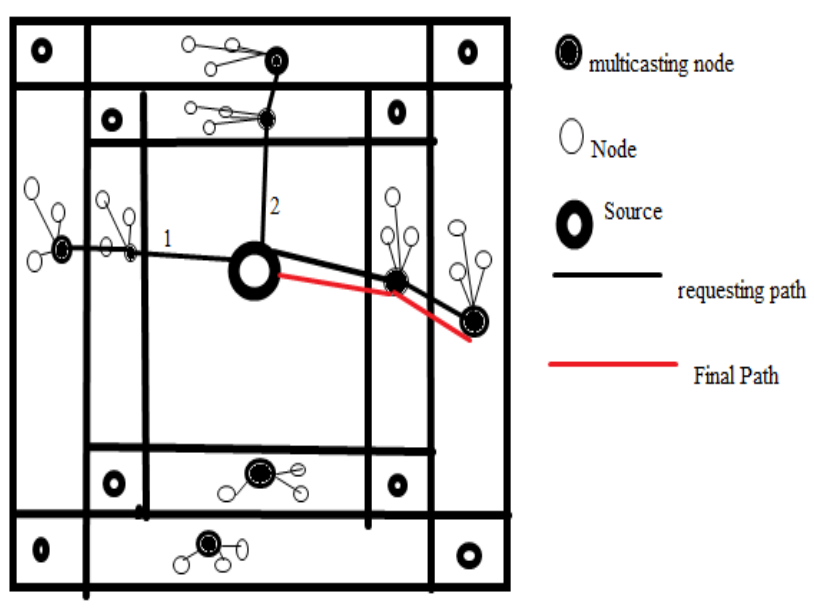

Fig 3: Multicasting Scenario

Both the protocol, i.e. AODV and DSR use the flooding approach for the route establishment. The route request messages are flooded into the network, and intermediate nodes which are having the path to destination will reply back with the route reply packets. The source selects the best path on the basis of Hop count and sequence number. In the approach of flooding, the network resources like bandwidth, node energy will get wasted. To overcome the problem, of flooding approach will be replaced with the multicasting approach. In this work, we mainly focus on to embed the approach of multicasting in AODV protocol for route establishment between source and destination.

\section{RESULTS}

We have used following simulations to study and analyze our result. They are NS2 network simulator, NAM editor to show the simulations schemes for the multicasting in AODV protocol and their performances and their routing paths. Furthermore we have used X-graph to graphically represent the number of packets loss, throughput and delay in transmission of packets. we have perform the simulations on 41 number of mobile nodes, wireless channel type is used with Omni directional antenna type.

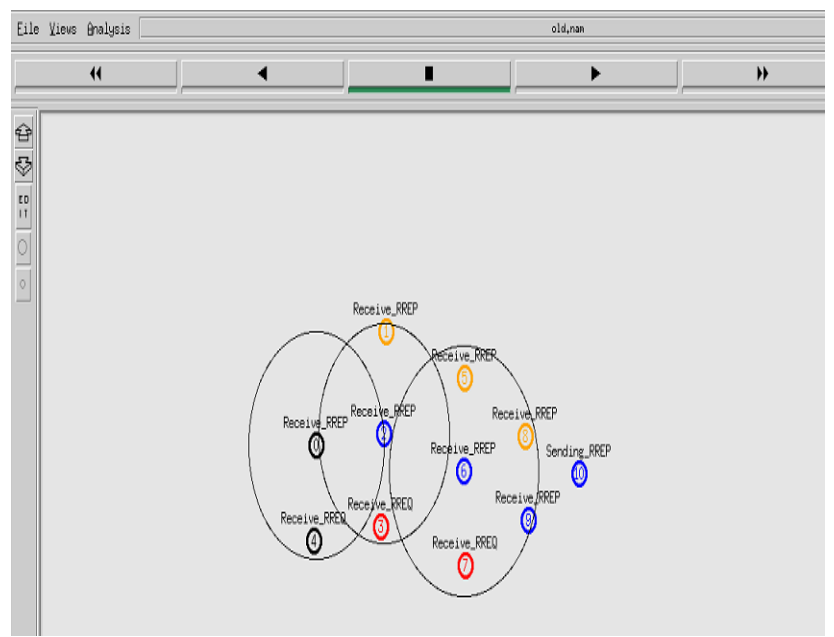

Fig 4: Simulation representation of AODV 
(See Figure 4). It shows that flooding approach is used in aodv protocol. Nodes of orange and red colour shows that they are sending RREQ packets and nodes of blue colour shows that they reply with RREP packets with having shortest hop-count. (see Figure 5). New scenario of multicasting in aodv protocol shows that adjacent multicasting node which is having direct path to destination will respond back to source with the route reply packets.

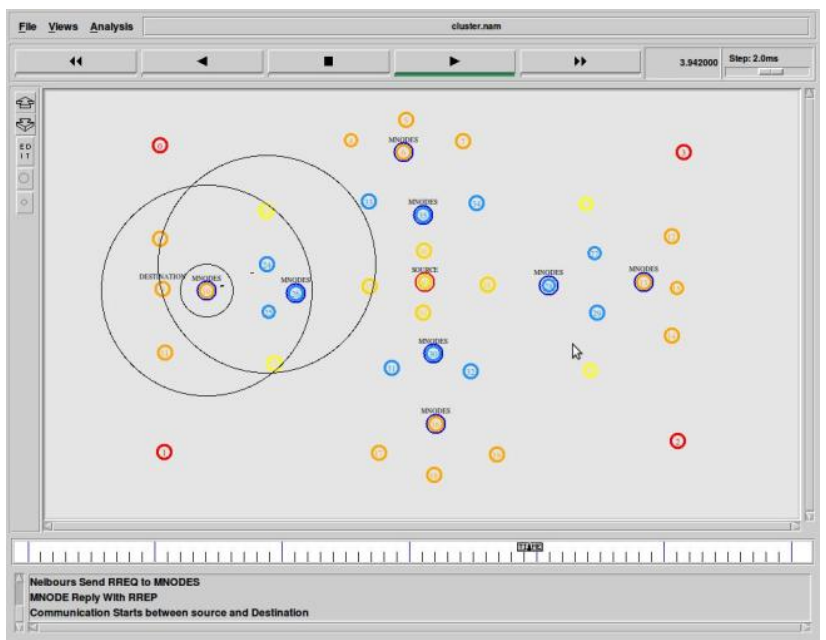

Fig 5: Simulation representation of new scenario.

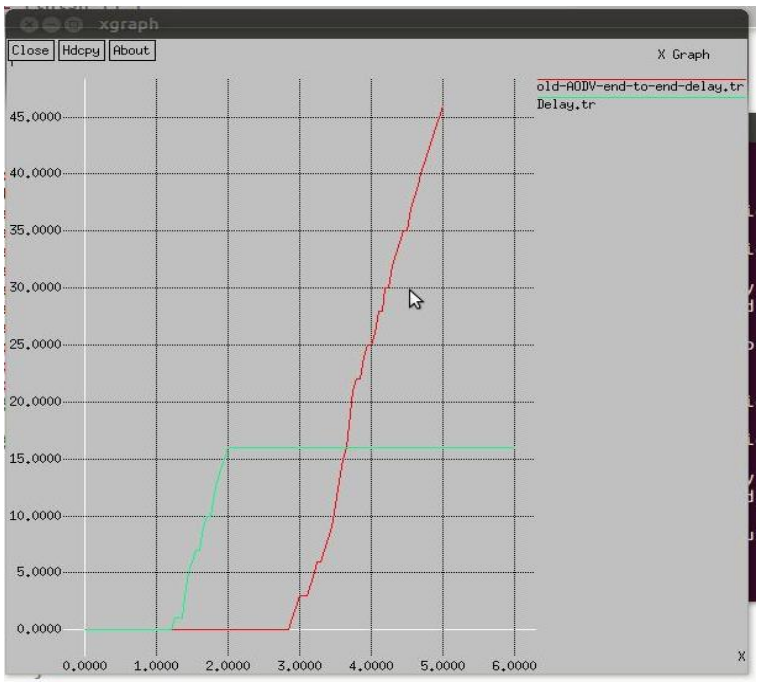

Fig 6: Graphically representation of Delay

(see Figure 6). the red line shows the delay in aodv without using multicasting and green line shows the new scenario delay. The delay in the new scenario is less due to effective and efficiently path establishment between source and destination. (see Figure 7). the green line shows the packet loss in the new scenario and red line shows the packet loss in the old scenario. The packet loss in the old aodv scenario is more due to the broadcasting technique of tradition AODV protocol.

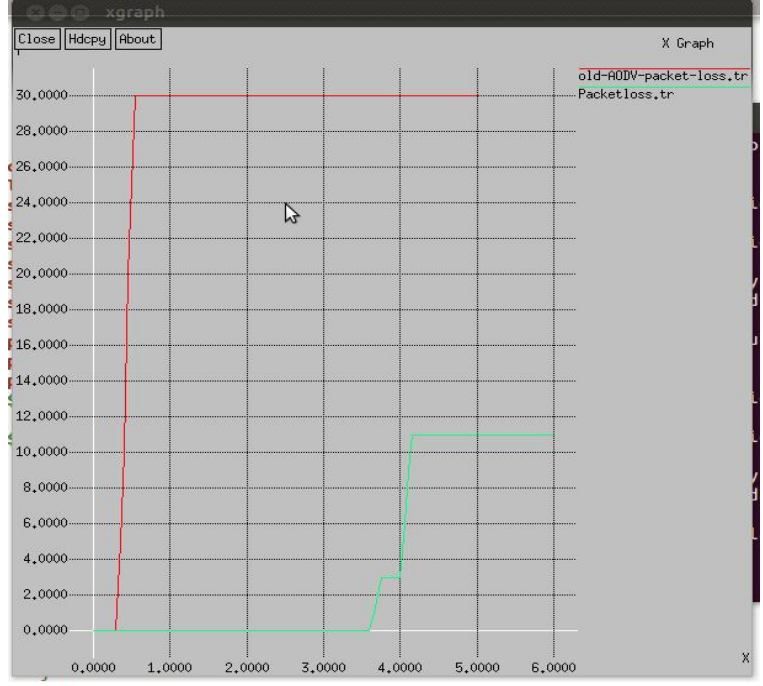

Fig 7: Graphical representation of Packet Loss

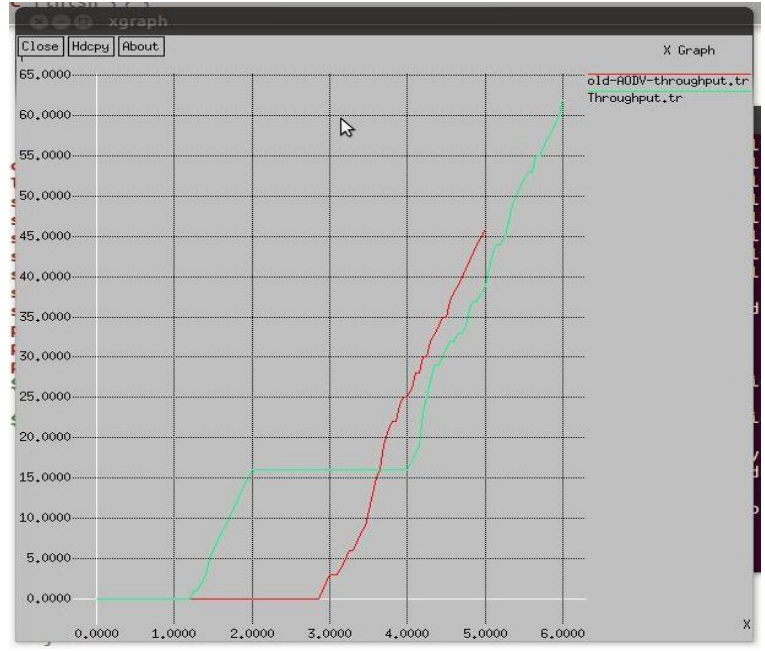

Fig 8: Graphically representation of throughput

(See Figure 8) The green line shows the throughput of the new scenario and red line shows the throughput of the old scenario. The throughput of the new scenario is more as compared to the old scenario. This is due to less number of nodes takes participate in multicasting than broadcasting and easy route establishment in between source and destination.

\section{CONCLUSION}

AODV protocol is easier to implement and to integrate with various other mechanisms. AODV protocol has better scalability. Aodv uses flooding approach for finding path from source to destination and AODV maintains only one route per destination. This is one of the major problems in AODV, since every time a route is broken; a route discovery has to be initiated. This leads to more overhead, higher delays and high packet lost.. In this paper we focused on tree formation from source to destination using multicasting nodes. We believe that proposed algorithms discussed in this paper will give benefit for various research scholars. Its experimental results show that proposed technique gives better result which has better throughput and less packet loss and less delay as compare to traditional aodv protocol. 


\section{ACKNOWLEDGEMENTS}

Special Thanks to all the supported staff and also thankful my Guide Mr. Mritunjay Kumar Rai.

\section{REFERENCES}

[1] Abdul Haimid, Bashir Mohamed, Analysis And Simulation Of Wireless Ad-Hoc Network Routing Protocols, 2004.

[2] Sunil Taneja, Ashwani Kush, Amandeep Makkar, End to End Delay Analysis of Prominent On-demand Routing Protocols, IJCST Vol. 2, Issue 1, 2011.

[3] Giovanni Vigna, Sumit Gwalani, Kavitha Srinivasan, Elizabeth M., Royer Richard, A. Kemmerer, "An Intrusion Detection Tool for AODV-based Ad hoc Wireless Networks" 2004.

[4] Humayun Bakht, et al. "Survey of Routing Protocols for Mobile Ad-hoc Network", International Journal of Information and Communication Technology Research. Volume 1 No. 6, ISSN-2223-4985 2011.

[5] R.Balakrishna, et al. "Performance Issues On Aodv and Dsdv For Manets", Journal Of Theoretical And Applied Information Technology.

[6] Sevil Şen, John A. Clark, Juan E. Tapiador, "Security Threats in Mobile Ad Hoc Networks" 2010.
[7] A.A. Kharazian, K. Jamshidi and M.R. Khayyambashi, "Adaptive clustering in wireless sensor network: considering nodes with lowest energy ", International Journal of Ad hoc, Sensor \& Ubiquitous Computing (IJASUC) Vol.3, No.2, April 2012.

[8] K. Maraiya, K. Kant, N. Gupta "Efficient Cluster Head Selection Scheme for Data Aggregation in Wireless Sensor Network" International Journal of Computer Applications, Volume 23-No. 9, June 2011.

[9] Vinit Garg, Manoj Kr.Shukla, Tanupriya Choudhury, Charu Gupta, "Advance Survey of Mobile Ad-Hoc Network," IJCST Vol. 2, Iss ue 4, Oct. Dec. 2011

[10] Amit N. Thakare Mrs. M. Y. Joshi "Performance Analysis of AODV \& DSR Routing Protocol in Mobile Ad hoc Networks" IJCA Special Issue on "Mobile Ad-hoc Networks" MANETs, 2010

[11] Mina Vajed Khiavi, "Performance Comparison of AODV, DSDV, DSR and TORA Routing Protocols in MANETs" International Research Journal of Applied and Basic Sciences. Vol., 3 (7), 1429-1436, 2012

[12] Sahil Gupta, "Simulation Based Performance Comparison of AODV and DSR Routing Protocols in MANETS", International Journal of Applied Engineering Research, ISSN 0973-4562 Vol.7 No.11 (2012) 